\title{
Addendum to the Acknowledgments: Comparison of Intercom and Megaphone Hashtags Using Four Years of Tweets From the Top 44 Schools of Nursing: Thematic Analysis
}

Kimberly Acquaviva, MSW, PhD, CSE, FNAP

School of Nursing, University of Virginia, Charlottesville, VA, United States

Corresponding Author:

Kimberly Acquaviva, MSW, PhD, CSE, FNAP

School of Nursing

University of Virginia

4005 McLeod Hall

Charlottesville, VA, 22903

United States

Phone: 12024230984

Email: kda8xj@virginia.edu

\section{Related Article:}

Correction of: https://nursing.jmir.org/2021/2/e25114

(JMIR Nursing 2021;4(2):e29823) doi: 10.2196/29823

In "Comparison of Intercom and Megaphone Hashtags Using Four Years of Tweets From the Top 44 Schools of Nursing: Thematic Analysis" (JMIR Nursing 2021;4(2):e25114 doi: $10.2196 / 25114$ ), the author noted one error.

In the originally published paper, the Acknowledgments section contained the following line about the source of funds for the 2016-2018 Twitter data:

The September 29, 2016, to February 22, 2018, Twitter data for this project were purchased with funds provided by the George Washington University School of Nursing's Center for Health Policy and Media Engagement.

To increase clarity regarding the amount and original sources of funding provided for the purchase of data, this has been corrected to:

The September 29, 2016, to February 22, 2018,

Twitter data for this project were purchased by the
George Washington University School of Nursing's Center for Health Policy and Media Engagement for $\$ 1000$ with funds received from the Gordon and Betty Moore Foundation, Robert Wood Johnson Foundation, Beatrice Renfield Foundation, Sigma Theta Tau International, American Association of Critical-Care Nurses, Donald and Barbara Jonas Foundation, National League for Nursing, OnCourse Learning, American Association of Colleges of Nursing, American Organization of Nurse Executives, and Wolters Kluwer Health. No funding was provided for this study beyond the $\$ 1000$ used for the purchase of data.

The correction will appear in the online version of the paper on the JMIR website on April 29, 2021, together with the publication of this correction notice. Because this was made after submission to PubMed, PubMed Central, and other full-text repositories, the corrected article has also been resubmitted to those repositories.

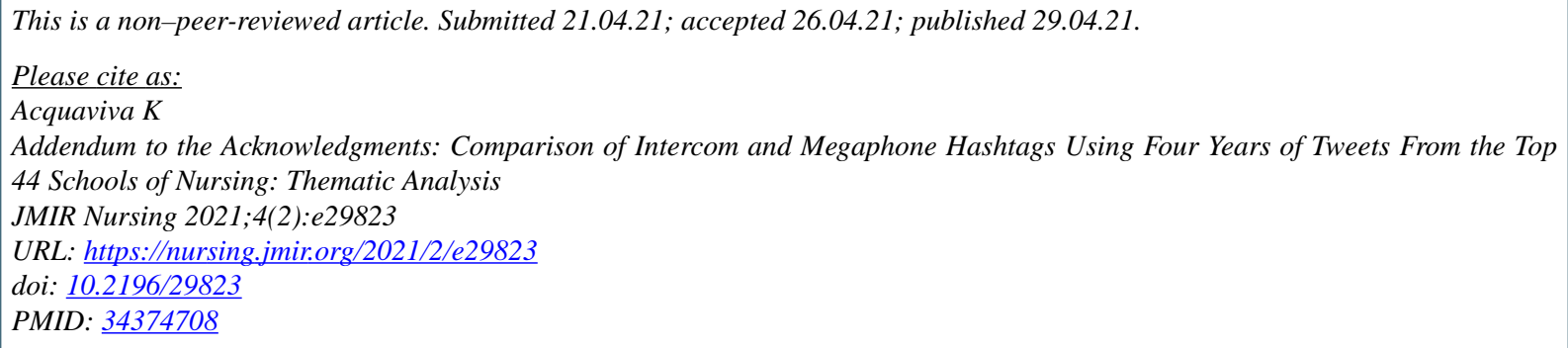


CKimberly Acquaviva. Originally published in JMIR Nursing (https://nursing.jmir.org), 29.04.2021. This is an open-access article distributed under the terms of the Creative Commons Attribution License (https://creativecommons.org/licenses/by/4.0/), which permits unrestricted use, distribution, and reproduction in any medium, provided the original work, first published in JMIR Nursing, is properly cited. The complete bibliographic information, a link to the original publication on https://nursing.jmir.org/, as well as this copyright and license information must be included. 